\title{
Potential benefits of dosimetric VMAT tracking verified with 3D film measurements
}

\author{
Crijns Wouter[a][b], Defraene Gilles[a][c], Van Herck Hans[c][d], Depuydt Tom[a][b], \\ Haustermans Karin[a][b], Maes Frederik[c][d][e], Van den Heuvel Frank[f]
}

5 [a] KU Leuven Department of Oncology, Laboratory of Experimental Radiotherapy, Herestraat 49, 3000 Leuven, Belgium

[b] Radiation Oncology, University Hospitals Leuven, Herestraat 49, 3000 Leuven

[c] KU Leuven Medical Imaging Research Center, Herestraat 49, 3000 Leuven,

Belgium

10 [d] KU Leuven Department of Electrical Engineering (ESAT) - PSI, Center for

Processing Speech \& Images, 3000 Leuven, Belgium

[e] KU Leuven iMinds - Medical IT department, 3000 Leuven, Belgium

[f] University of Oxford, Department of Oncology, MRC-CR-UK Gray Institute of

Radiation Oncology and Biology, Oxford OX1 2JD, United Kingdom

\begin{abstract}
15Abstract
Purpose: To assess the impact of realistic intra-fraction prostate motion on both focal boost and hypo-fractionated prostate VMAT plans, using 3D film-stack dose measurements for different plan adaptation strategies: our current clinical practice (a couch shift), geometric tracking, and dosimetric tracking.
\end{abstract}

20Methods and Materials: A focal boost (35x2.2 Gy and $35 \times 2.7 \mathrm{~Gy}$ ) and a hypo-fractionated ( $5 \times 7.25 \mathrm{~Gy}$ ) prostate VMAT plan were created for a heterogeneous phantom with internal prostate motion. For these plans geometric tracking and dosimetric tracking were measured by ionization chamber (IC) point measurements (zero-D) and a measurements using a stack of EBT3 films (3D). Geometric tracking applied translations, rotations and scaling of the MLC aperture in response to 25realistic prostate motions. The dosimetric tracking additionally corrected the monitor units to resolve variations due to difference in depth, tissue heterogeneity, and MLC-aperture. The tracking was based on the positions of four fiducial points only.

The film measurements were compared to the gold standard (i.e. IC measurements) and the planned dose distribution. Additionally, the 3D measurements were converted to dose volume histograms,

30 tumor control probability and normal tissue complication probability parameters (DVH/TCP/NTCP) as a direct estimate of clinical relevance of the proposed tracking. 
Results: Compared to the planned dose distribution, measurements without prostate motion and tracking showed already a reduced homogeneity of the dose distribution. Adding prostate motion further blurs the DVHs for all treatment approaches. The clinical practice (no tracking) delivered the 35 dose distribution away from its intended position with boost dose errors up to $10 \%$, but still inside the CTV to PTV margins. The geometric and dosimetric tracking corrected the dose distribution's position. Moreover, the dosimetric tracking could achieve the planned boost DVH, but not the DVH of the more homogeneously irradiated prostate. A drawback of both the geometric and dosimetric tracking was a reduced MLC blocking caused by the rotational component of the MLC aperture corrections.

40Because of the used CTV to PTV margins and the high doses in the considered fractionation schemes, the TCP differed less than 0.02 from the planned value for all targets and all correction methods. The rectal NTCP constraints however could not be realized using any of the methods.

Conclusions: Geometric and dosimetric tracking with a limited input deposits the dose distribution with higher positional accuracy than the clinical practice. The latter case has boost dose errors up to $4510 \%$. The increased accuracy has a modest impact $[\Delta(\mathrm{NT}) \mathrm{CP}<0.02]$ because of the applied CTV margins and the use of high doses. To allow further margin reduction tracking methods are vital. The proposed methodology could further be improved by implementing a rotational correction as collimator rotation rather than changing a MLC aperture. 


\section{Introduction}

Real-time tumor tracking for motion management in radiotherapy has the potential to increase the precision of the dose deposition by continuously redirecting the beam to the new target position. Tracking is currently becoming available on various treatment units $^{1-4}$. For prostate the clinical relevance of tracking might be limited in classic 55fractionation schemes, e.g. more than 20 fractions delivering a uniform dose to the prostate. Dose reconstructions pointed out that clinical relevant errors average out after 8 IMRT fractions depositing a uniform 3.2 Gy dose ${ }^{5}$ and similarly after 5 fractions of 2 Gy for Tomotherapy ${ }^{6}$ and Intensity Modulated Radiotherapy ${ }^{7,8}$ (IMRT).

This number of fractions is currently reduced using hypo-fractionation [e.g. 5 fractions 60of 7.25 Gy (RTOG 0938)] and stereotactic body radiotherapy (SBRT) ${ }^{9-12}$. The goal of the reduced fraction numbers is an increase of the target dose without an increase of the normal tissue toxicity ${ }^{12}$. Additional advantages are: a reduced total time spend on the treatment unit, which increases the patients' comfort; and a potential treatment costs reduction ${ }^{9}$. But with only a few fractions applied, the intra-fraction motion becomes 65 more relevant even for uniform dose distributions ${ }^{5-8}$. Moreover, the modern prostate treatments use a non-uniform dose distribution to apply an ablative focal boost to a suspicious region inside the prostate ${ }^{13}$. Such dose distributions are not yet studied in combination with intra-fraction prostate motions.

Current Such dose distributions are typically delivered with modulated treatment 70techniques: IMRT or Volumetric Modulated Arc Therapy (VMAT ${ }^{14}$ ). The combination of tracking with these modulated delivery techniques is currently evaluated using dose reconstructions of DMLC tracking for prostate ${ }^{15}$ and for lung ${ }^{17}$. But this promising combination of tracking and modulated treatments is not yet clinically implemented.

The IMRT and VMAT These tracking methods ${ }^{15-17}$ as well as the clinically implemented 75tracking ${ }^{1-4}$ leave the nominal beam output (Monitor Units; MU) unaltered. This remaining degree of freedom can account for variations along the beam's path e.g. depth, and tissue heterogeneity variations. The present is work explores the potential of dosimetric tracking of prostate intra-fraction motion during modulated VMAT treatments. 
80The integration of geometric tracking with modulated treatments has mainly been investigated using dose reconstructions $s^{5,15,18}$. Dose measurements of motion managed modulated radiotherapy have previously been performed for prostate in a moving rigid phantom ${ }^{17,19,20}$ but not with a moving prostate volume inside a phantom. The latter seems to be important as tracking is used to cope with internal organ motion rather 85than total body motion. Here a heterogeneous prostate phantom with internal phantom motion is used to measure the dose deposited by the proposed dosimetric tracking using a stack of 2D films i.e. an anisotropic 3D measurement. This exploratory study used a synchronization of the motion phantom and the treatment unit to bypass a realtime implementation.

90Therefore, the current work allowed to study the effect of different realistic prostate motions ${ }^{21}$ on both a hypo-fractionated and a focal boost VMAT treatment using 3D dose measurements in a heterogeneous prostate motion phantom. The 3D measurements can be clinically interpreted by calculating the tumor control probability and the normal tissue complication probability (TCP and NTCP). 


\section{Methods and Materials}

\section{A. Treatment plans and adaptation}

\section{A.1. Focal boost}

The first treatment plan is the plan proposed in the study arm of the Flame-trial ${ }^{13}$. The plan delivered $35 \times 2.2$ Gy to the entire prostate and aimed to deliver $35 \times 2.7$ Gy to a 100small boost region using a simultaneous integrated boost technique. The plan consists of a clockwise and counter clockwise $10 \mathrm{MV}$ RapidArc ${ }^{\circledR}$ beam with respective collimator angles of $10^{\circ}$ and $80^{\circ}$ (arc CW10 and arc CCW80). The treatment plans were created in the Eclipse $10.028^{\mathrm{a}}$ treatment planning system (TPS) for a TrueBeam ${ }^{\mathrm{TM}}$ STx equipped with a High Definition 120 MLC $^{a}$. The same equipment was used for a second treatment 105 plan.

\section{A.2. Hypo-fractionation}

The second treatment plan was based on the RTOG 0938 study arm which aims to study the delivery of an accelerated treatment with a uniform prostate dose: 5x7.25 Gy. The plan consists out of four alternating clockwise and counter clockwise 10MV RapidArc ${ }^{\circledR}$ 110beams, with respective collimator angles of $350^{\circ}, 10^{\circ}, 80^{\circ}$, and $110^{\circ}$.

\section{A.3. Treatment approaches}

Four scenarios were evaluated: a reference measurement without tracking nor internal phantom motion (Static) and three treatment approaches (with internal phantom motion): 1) The current clinical practice, which had no intra-fractional corrections after 115an initial positioning based on laser alignment and couch displacements-and a-_aser alignment(Clinical); 2) An experimental simulation of a geometric tracking approach, which applied multi-leaf-collimator corrections only (MLC tracking); And 3) an experimental simulation of dosimetric tracking, which applied both MLC and MU corrections (MLC+MU tracking).

120The tracking methods are based on a forward planning approach to correct the MLC positions in the BEV, in combination with point dose calculations along the beam's path to alter the beam's output (MU). The corrections were executed per control point as summarized below. A detailed description can be found in Ref ${ }^{22}$.

a Varian Medical Systems, Palo Alto, California, USA 


\section{Geometric or MLC tracking}

$125 \mathrm{New}$ MLC positions were calculated for each control point (CP) from the projections of four fiducial points in the Beam's Eye View (BEV). The MLC-corrections included scaling and rotations in addition to the classic translations. To fully explore the benefits of dosimetric tracking an ideal marker detection was assumed: the exact marker positions were considered to be known at any time point (i.e. real-time), see section 2.B. The 130actual marker detection was outside the scope of this work.

\section{Dosimetric or MLC + MU tracking}

The MU-weight was corrected per CP using point dose calculations towards the four fiducial points. The point dose calculations relied on a pre-treatment (CB)CT ${ }^{23}$ (i.e. online) and the real-time positions of the fiducial points. A more detailed description of 135the VMAT adaptation can be found elsewhere ${ }^{22,23}$. A difference with the previously published methods is that the maximal leaf-speed of the new corrected plans was limited to the maximal leaf speed of the initially optimized plan.

\section{B. Experimental Setup}

\section{B.1. Heterogeneous phantom with internal motion}

140The Dynamic Pelvis Phantom ${ }^{b}$ was used for both treatment planning and dose measurements. The phantom itself was tissue equivalent apart from the bony anatomy. A rotating disk (Figure 1) allowed a coupled longitudinal and vertical prostate motion. The phantom has two synchronized motion drivers, both positioned outside the treatment field (not depicted in Figure 1).

145The phantom's rotating disk has a cubic cut-out to hold dedicated inserts such as: an ionization chamber (IC)-insert, a radiological insert used for prostate delineation, or a film stack for 3D-dose measurements. For this reason the measurement volume is restricted to the dimensions of the film-insert. All reported analyses were limited to this volume of interest (VOI, $63 \times 63 \times 63 \mathrm{~mm}^{3}$ ), see Figure 1.

b CIRS, Virginia, USA 

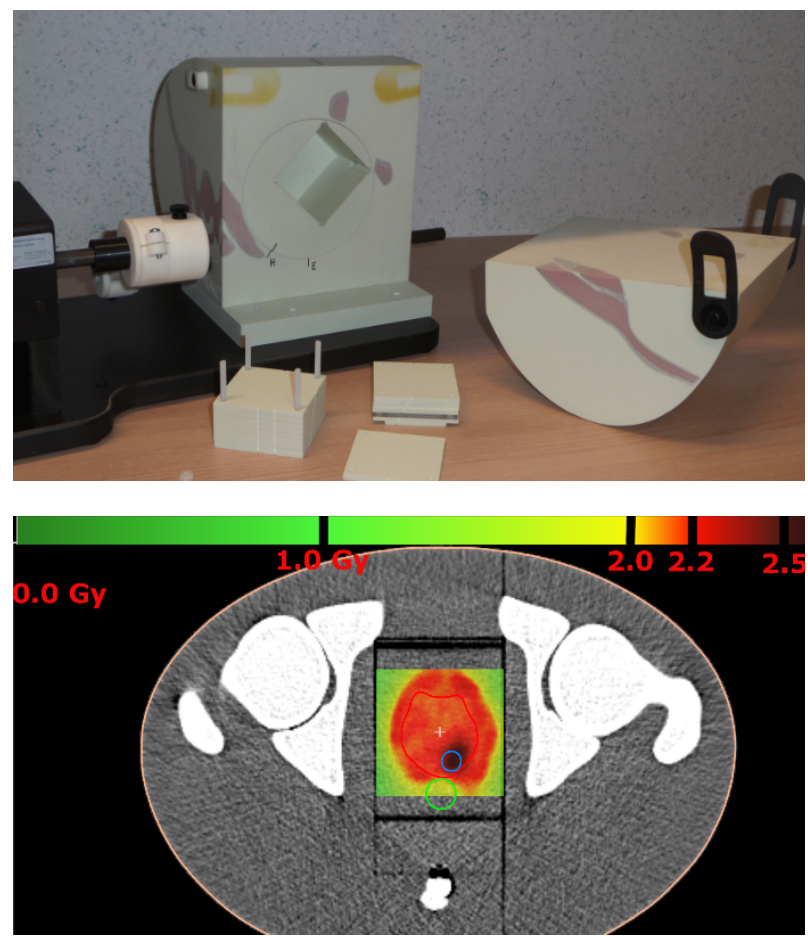

150Figure 1: The upper panel shows the pelvic motion phantom, with the disassembled film stack in the lower part of the picture. The lower panel contains an axial plane depicting the planning structures: CTV (red), boost volume (blue), rectum (green); as well as the calculated dose distribution within the (VOI) i.e. the measurement volume. Only part of the rectal structure is included in this VOI. DVHs' throughout this manuscript are limited to this VOI.

\section{B.2. Synchronization}

The two motion drivers were used to synchronize the internal phantom motion and the treatment unit's beam on. This procedure bypassed the need for a real-_time implementation (of dosimetric tracking) on our treatment unit. The first motion driver rotated the disk simulating a realistic prostate motion. The second motion driver applied 160a square wave to the Real-time Position Management ${ }^{\mathrm{TM}}$ (RPM ${ }^{\mathrm{c}}$ ) block. The square wave was detected through the RPM system and it initiated the irradiation when a predefined threshold was exceeded (Figure 2).

Consequently the experimental setup consisted of an offline adaptation using a priori known motion trajectories to adjust the initial treatment plan [Figure 2 (a), offline 165section]. The real-time part was driven by the motion phantom which applied both the internal phantom motion and the trigger to start the actual dose delivery [Figure 2 (a), Real-Time section].

c Varian Medical Systems, Palo Alto, California, USA 


\section{B.3. Prostate Motions}

Three typical prostate motions recorded by Langen et al. ${ }^{21}$ were applied by the Dynamic 170Pelvis Phantom: a drift motion (Drift), a drift with additional jumps (Jump), and a pattern with high frequency prostate jumps (HF), see Figure 2 b. The original Jump and HF motions were adjusted to the phantom limits, i.e. the amplitudes were scaled by a factor 0.58 (Jumps) and 0.67 (HF) and the anterior posterior (Ant-Post) baseline was corrected by $-4.40 \mathrm{~mm}$ (Jumps) and $-5.79 \mathrm{~mm}$ (HF). The resulting motions are depicted 175in Figure 2 b. The same figure contains an indication of the beam-on of the different arcs. 


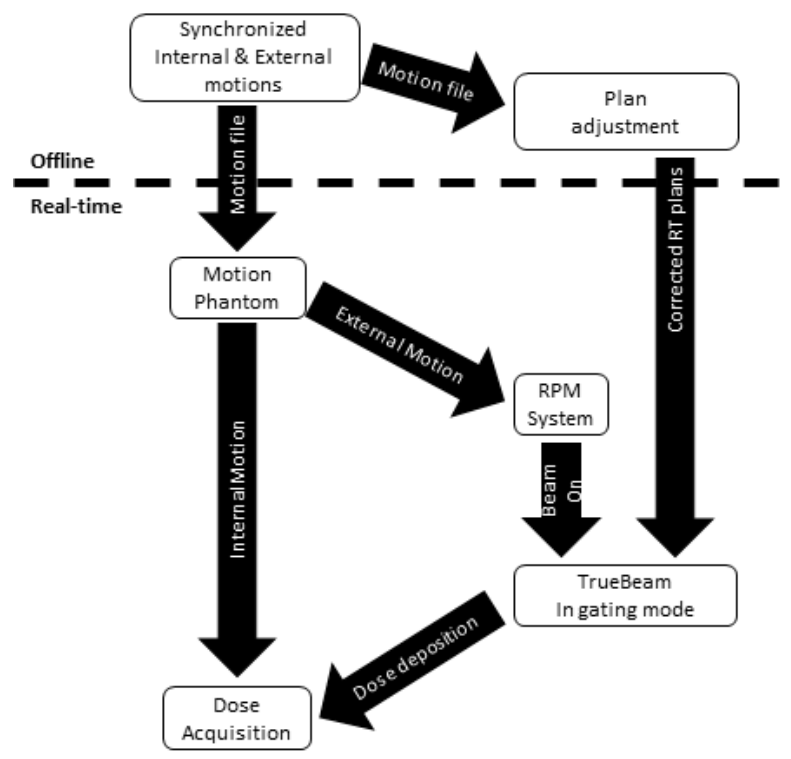

a)

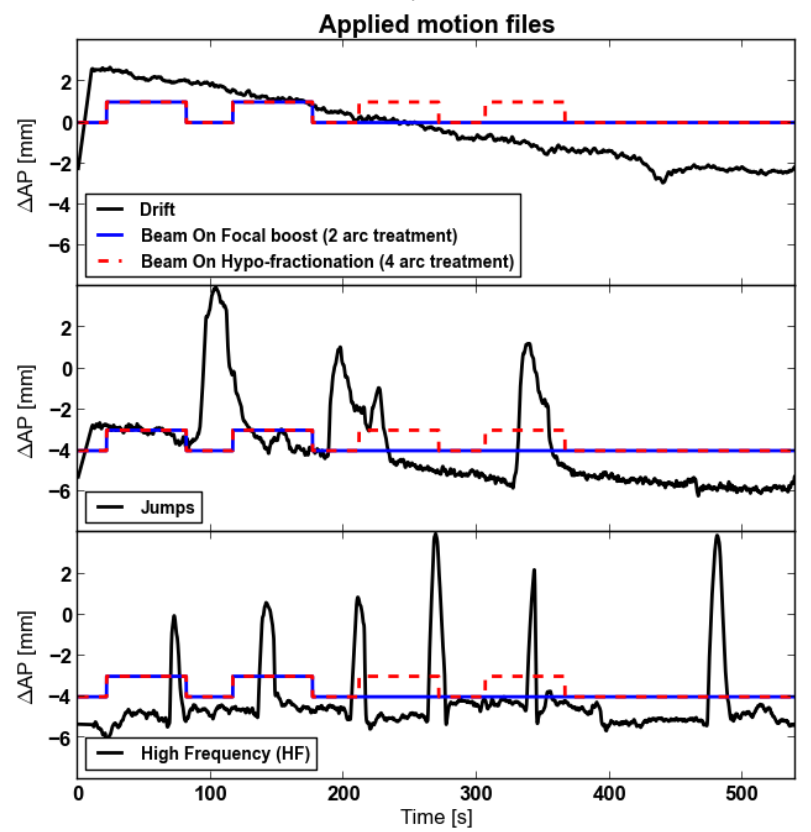

b)

Figure 2: The upper panel contains a flow chart of the experimental setup, with an offline plan correction in preparation of the real-time synchronized prostate motion and dose deposition. The lower panel depicts the anterior posterior prostate motions applied to the pelvic phantom. The $y$-axis depicts 180anterior posterior displacement for the prostate's center off mass according to the treatment planning position. The block waves indicating the beam-on time for the two treatment types are indicated in color: focal boost (blue) and hypo-fractionation (red). 


\section{B.4. Treatment planning and plan corrections}

The prostate as visible in the radiological insert was cropped with a uniform $2 \mathrm{~mm}$ 185 margin to guarantee that all planned dose gradients are inside the VOI. The resulting volume defined the clinical target volume (CTV or prostate; $32.3 \mathrm{cc}$ ). A spherical focal boost volume (boost; $0.4 \mathrm{cc}$ ) and a cylindrical rectal volume $(10.78 \mathrm{cc})$ were ad hoc defined (Figure 1). The rectum emulated a realistic treatment planning situation with sparing of an organ at risk in close proximity. The VOI included only a part of the 190rectum $(4.91 \mathrm{cc})$.

A planning target volume was defined using the margins prescribed in the Flame-trial ${ }^{13}$ : the boost volume was expanded with a uniform $8 \mathrm{~mm}$ margin, the CTV with $4 \mathrm{~mm}$, the union of these two volume minus the rectal volume forms the PTV i.e. $P T V=[($ Boost $+8 \mathrm{~mm}) \cup(C T V+4 \mathrm{~mm})]-$ Rectum. The treatment plans aimed to deliver 195at least $35 \times 2.2 \mathrm{~Gy}$ (focal boost) or $5 \times 7.25 \mathrm{~Gy}$ (hypo-fractionation) to this PTV $(61.43 \mathrm{cc})$. To guarantee that the MLC-apertures remained in the treatment field during tracking, a $17 \mathrm{~mm}$ large jaw-to-PTV outline distance was used.

An in-house application corrected the treatment plans in preparation of the experiments. The application's input was an a priori known motion pattern and a dicom 200export of the initial treatment plan. The corrected dicom plans were imported in the planning system and calculated, which is necessary for a subsequent delivery. In the TPS, MU and field-size changes were kept invariant during this calculation. Subsequently, the treatment plans could follow the clinical workflow.

\section{C. 3D film dosimetry}

205For each of the prostate motions (Drift, Jumps, and HF), an IC and an EBT3 film measurement was performed for each of the treatment approaches (Clinical, MLC, and $M L C+M U)$ and for both the focal boost and the hypo-fractionated treatment. Additionally, the dose deposition of the initial treatment plan was measured without target motion (Static). The IC measurements were performed per arc. The EBT3 film 210measurements were performed per treatment plan. The films were calibrated as described previously ${ }^{24}$. A detailed description of the film stack measurements can be found in the supplemental material Film measurements. 
Point measurements were performed using a pinpoint IC ${ }^{d}$, positioned in the center of the prostate using a dedicated insert. The point measurements were compared to the 215calculated point doses of the TPS, and to the corresponding position in the 3D film measurements, average of a $0.7 \times 0.7 \mathrm{~mm}^{2}$ area on two succeeding film layers ( $4 \times 4$ pixels area on two layers). Some of these per arc IC-measurements showed large deviations with the planned values. The arc with the largest deviations was individually measured on film for all adaptation approaches (Static, Clinical, MLC, and MLC+MU) to investigate 220the cause of the deviations. All other film measurements were per treatment plan, not per arc.

3D film measurements were performed using a dedicated film insert allowing 2D measurements for ten planes $\left(63 \times 63 \mathrm{~mm}^{3}\right)$. The in-plane resolution was 0.169 $\mathrm{mm} /$ voxel, and the plane separation equaled $4.6 \mathrm{~mm}$. The zero-position of the rotating 225 measurement's coordinate systems has an angle of $46^{\circ}$ compared to the TPS-coordinate system. Figure 1 illustrates the zero-position of the rotating disk. For comparisons, the smooth TPS dose distributions (1x1x1mm/voxel) were reformatted towards the measurement's coordinate system and only the planes corresponding to the measurement planes were retained using a tri-linear interpolation. This operation was 230performed in MeVisLab ${ }^{e}$ and the resulting calculated dose planes were considered as the 'planned' dose in the analysis below. The difference between the resulting planned DVH's and the initial TPS-DVH's for the rectum and the boost volume are indicated in the supplementary material. Note that all comparisons presented in the work were based on dose distributions in the same coordinates system and with the same 235 resolution.

\section{D. Estimating the clinical impact}

The homogeneity of the dose distributions was quantitatively evaluated using the homogeneity index ( $\left.H I=\frac{D_{95 C T V}}{D_{5 C T V}}\right)$, and DVHs. The calculated and measured dose distributions were converted to DVHs for the prostate, the boost volume, the rectum, 240and the PTV, which was later considered as a zero margin CTV. For each of the

d PTW-Freiburg, Germany 
histograms the tumor control probability (TCP) or normal tissue complication probability (NTCP) were calculated using a biologic effective dose correction ( $a / \beta=3 \mathrm{~Gy}$ ).

The methodology of Webb and Nahum ${ }^{25}$ calculated the TCP with parameters from Oldham et al. ${ }^{26}$. For the focal boost treatment a clonogen density of $10^{5} \mathrm{~cm}^{-3}$ and $24510^{6} \mathrm{~cm}^{-3}$ was used for the prostate volume minus the boost volume and the boost

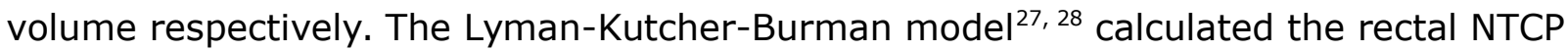
using the best estimate QUANTEC-parameters ${ }^{29}$.

TCP and NTCP were chosen as evaluation parameters because they weight the impact of the minimal, intermediate, and maximal doses of the considered organs in a clinically 250relevant way. These parameters were used for a comparison between different adaptation approaches rather than for their absolute values.
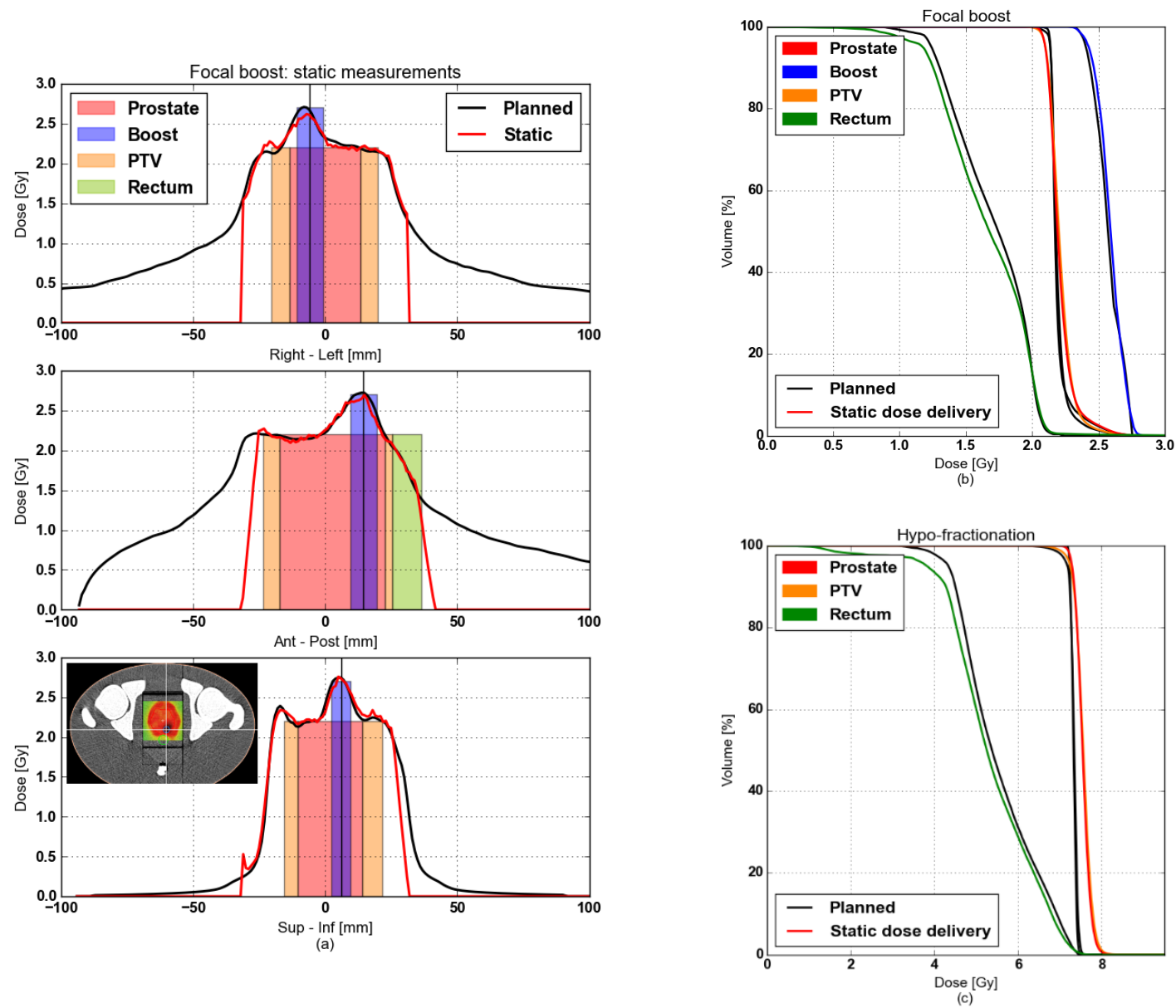

Figure 3: Left: dose profiles for the focal boost as planned (black) and as measured without phantom motion (static, red). The position of the dose profiles is indicated by the black line and corresponds with the white cross-hair in the inset, see also Figure $6 \mathrm{c}$. The position of the overlaid colored blocks 
255indicate the organ positions. The height of the Prostate, Boost, and PTV blocks corresponds to the prescribed dose, the height of the rectum block is arbitrary. Right: DVHs as planned (black) for both a focal boost treatment (upper panel) and a hypo-fractionated treatment (lower panel). DVHs were calculated using the VOI only, see Figure 1 . The colored lines depict the results of a static dose delivery. 


\section{Results}

\section{A. Static measurements}

With internal organ motions absent, measurements corresponded well to the TPS calculations: a correct position of the dose gradients (Figure 3) and point dose errors smaller than 3\% (Figure 4: focal boost: $\Delta D_{\text {static }}=-1.4 \%$ [IC], 0.5\% [Film]; hypo265fractionation: $\Delta \mathrm{D}_{\text {static }}=-1.2 \%$ [IC], 2.6\% [Film]). Nevertheless, the homogeneity of the dose distribution was reduced compared to the planned dose distribution $(\Delta \mathrm{HI}=-2.8 \%$ [focal boost] and $-4.3 \%$ [hypo-fractionation]).

The reduced homogeneity affected the CTV DVHs, but not the boost DVH (Figure 3). An overall decreased rectal dose resulted in slightly decreased complication probabilities 270(Figure 7: $\triangle \mathrm{NTCP}=-0.01$ [focal boost], -0.02 [hypo-fractionation]).
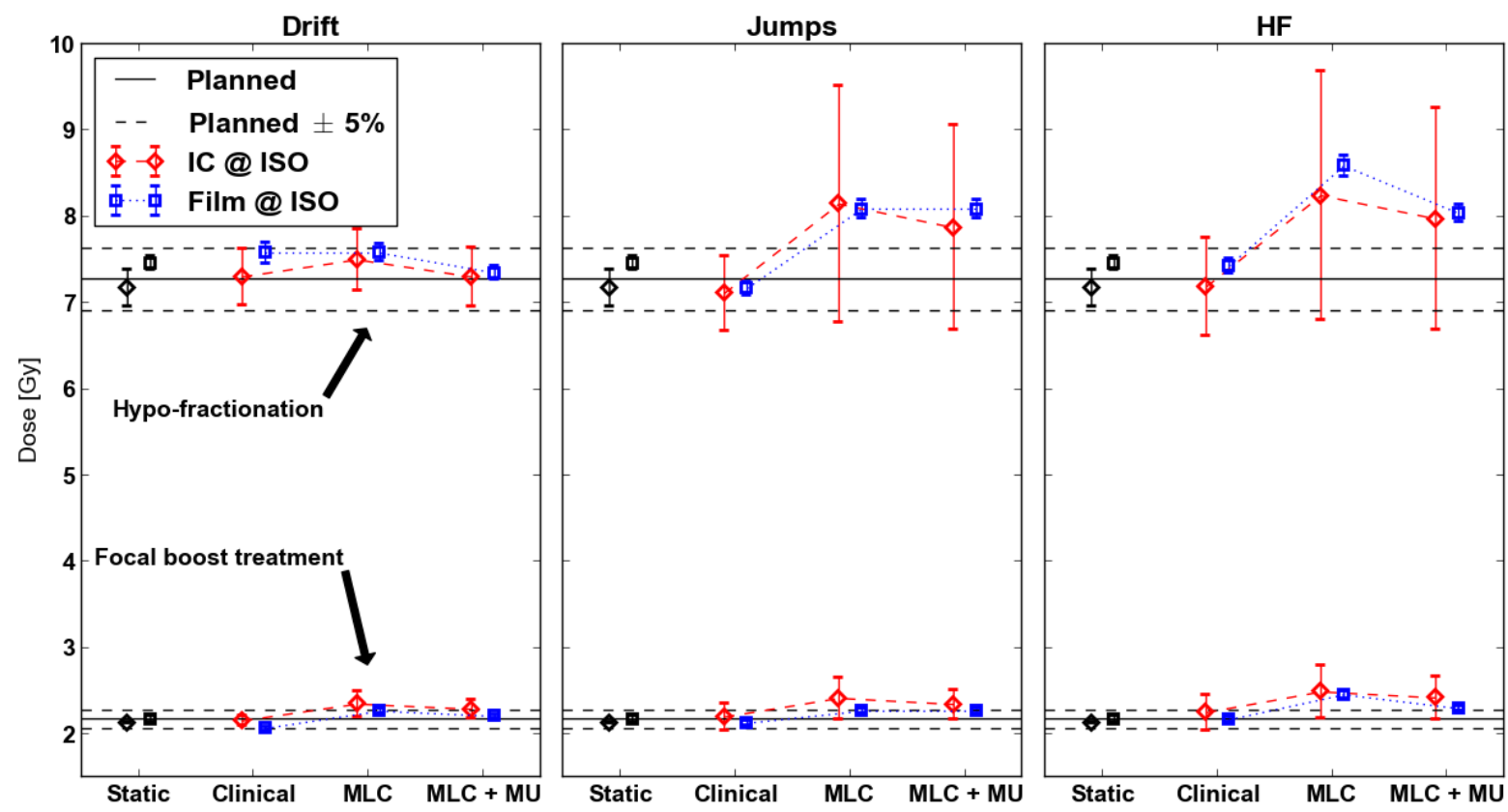

Figure 4: Point dose results in the center of the prostate using IC and film dose measurements of the complete treatment plans i.e. not per arc. The error bars are the mean error per plan (i.e. averaging over different arcs) for the IC measurements, and the standard deviation for the film measurements.

\section{B. Impact of prostate motion}

The point doses measured by both the IC and film (Figure 4) show the same pattern in function of the applied tracking and prostate motions. However, the per arc IC measurements had large deviations from the planned values. The largest deviations 
were observed for the CW10 arc in presence of the Jump motion: $\Delta D_{\text {IC }}=-4 \%$ (Static), $280-16 \%$ (Clinical), $+53 \%$ (MLC), and $+46 \%$ (MLC+MU). Per arc film measurements confirmed the IC results (Figure 5). The static dose measurement corresponded well to the planned dose distribution. The clinical practice shifted the dose distribution in the superior direction creating errors, which were in the order of 10 to $20 \%$ because of the integrated boost (Figure 4 and Figure 5). The tracking approaches managed to locate 285the dose at the planned position, but they did not reduce the dose centrally as was planned (Figure 5).

The profile and DVH results from the Jump motion were depicted as representative examples for all motions (Figure 6).

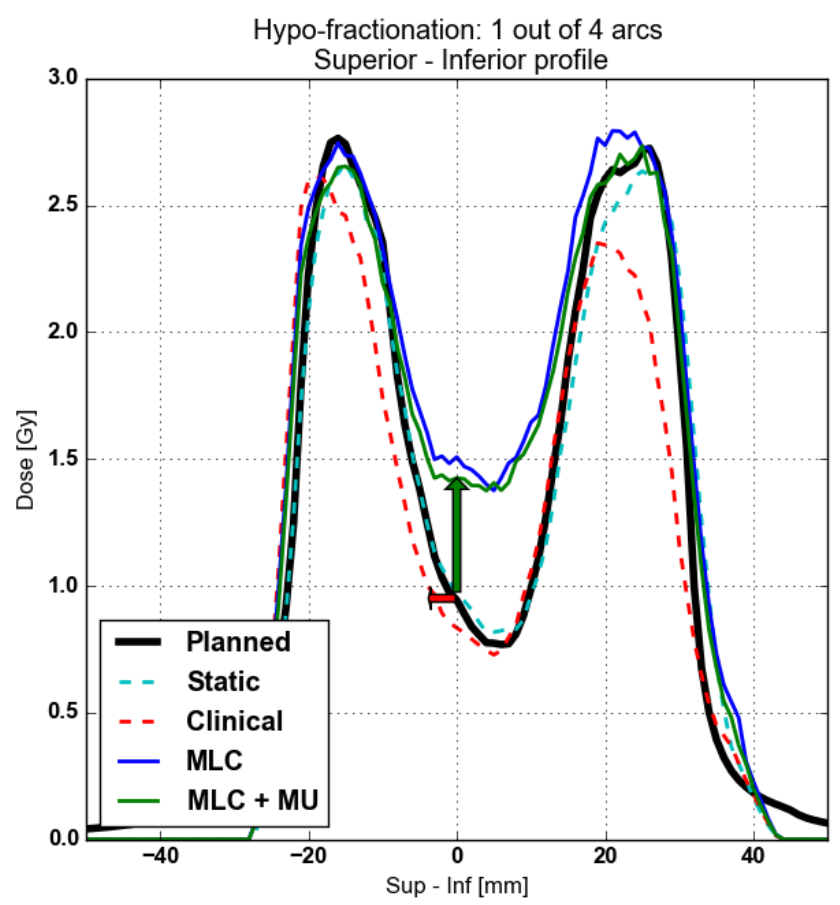

290Figure 5: Superior-Inferior dose profile through the isocenter of a per arc film measurement of the CW10 arc of the hypo-fractionation treatment in presence of Jump motions. This arc had the largest point dose deviations between IC and treatment planning. The clinical practice (red) has a positioning error (red arrow) i.e. the dose distribution is shifted superiorly. The tracking approaches corrected the dose distribution's position, but failed to reduce the central part of the dose distribution as planned 295(green arrow), this is attributed to a lack of MLC blocking imposed by the rotational correction of the MLC aperture, see Discussion. 


\section{B.1. Prostate drift motion}

Focal boost: $\quad$ For the focal boost the clinical practice deposited the dose distribution towards the rectum. The MLC and MLC+MU tracking were able to correct 300this position error (similar to the shift in Figure 6, but in the opposite direction). Nevertheless, all methods increased the rectal DVHs and NTCPs (Figure 7: $\triangle \mathrm{NTCP}=$ +0.12 [MLC], +0.07 [Clinical and MLC+MU]).

The MLC tracking delivered the intended boost dose, but increased the CTV dose, while the MLC+MU tracking improved the CTV dose, but decreased the boost dose. Both 305tracking approaches did not decrease the dose at the superior side of the boost as was planned (also observed for the Jump motion, see Figure 6). Consequently, all approaches further reduced the homogeneity compared to the planned value $(\Delta \mathrm{HI}=$ $-3.8 \%$ [Static], 5.6\% [Clinical], -7.3\% [MLC], -7.1\% [MLC+MU]) resulting in a larger blurring of the CTV DVHs. But the MLC+MU tracking resulted in the intended boost DVH. 310 Nevertheless, the impact on the TCP was negligible $\left(\Delta \mathrm{TCP}\right.$ Prostate $=-0.01[\text { Clinical }]_{,}<$ +0.01 [MLC, and MLC+MU], $\triangle \mathrm{TCP}_{\text {Boost }}<0.01$ ).

Hypo-fractionation: For a uniform hypo-fractionated dose distribution all approaches resulted in similar target doses with negligible TCP errors $(\leq 0.5 \%)$. Nevertheless, the target motion reduced the homogeneity $(\Delta \mathrm{HI}=-4.3 \%$ [Static], $-9.3 \%$ 315 [Clinical], $-7.5 \%[\mathrm{MLC}],-7.2 \%[\mathrm{MLC}+\mathrm{MU}])$. However, for the MLC+MU tracking the DVHs showed a slightly decreased dose for both the CTV and the rectum, while the clinical practice and the MLC only method had a slightly higher CTV dose but an increased rectum dose as well (Figure 7: $\triangle \mathrm{TCP}<0.01 ; \Delta \mathrm{NTCP}=-0.02$ [Static], +0.04 [Clinical], $-0.01[\mathrm{MLC}],-0.05[\mathrm{MLC}+\mathrm{MU}])$. 

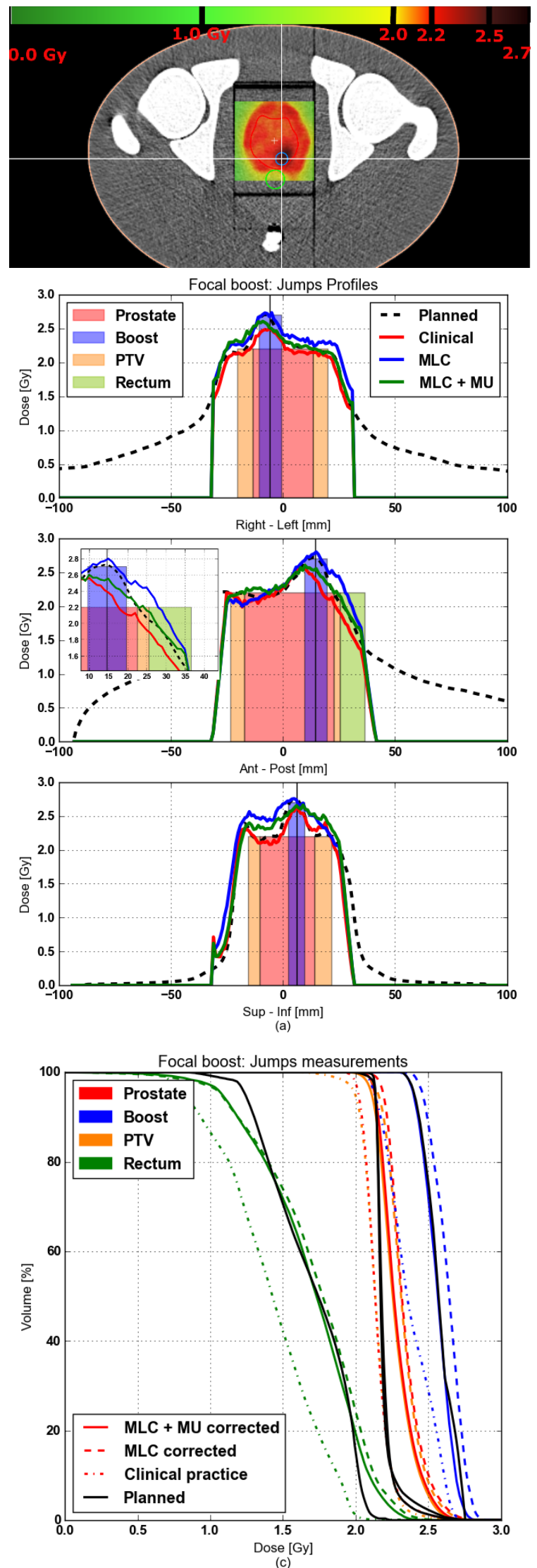
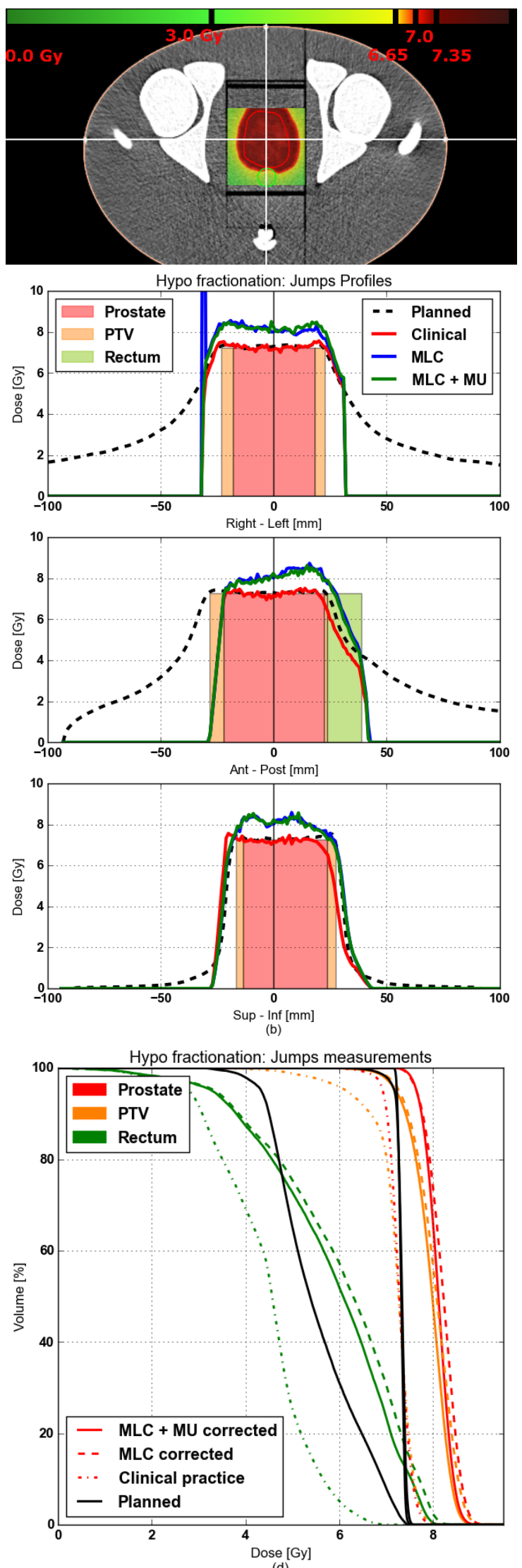
320Figure 6: Dose profiles ( $a, b)$ and DVHs ( $c, d)$ of the accumulated dose with prostate Jump motion. The profile position of the upper figures is indicated by the white cross-hairs in the axial dose planes on top. The position of the overlaid colored blocks indicate the organ positions. The height of the Prostate, Boost, and PTV blocks corresponds to the prescribed dose, the height of the rectum block is arbitrary. The clinical practice resulted in a positioning error for both the focal boost (left) and the hypo-

325 fractionated (right) treatment, mainly in the Ant-Post direction. The MLC and MLC+MU tracking corrected the positioning error. But the tracking approaches increased the dose superior of the focal boost volume. For the hypo-fractionation, the MLC and MLC+MU tracking had an increased dose for the Jump motion. Both over dosages were attributed to a lack of blocking, see Figure 8.

\section{B.2. Prostate jump motion}

330Focal boost: The clinical practice delivered the dose distribution with a shift away from the rectum. This was corrected by the MLC and MLC+MU tracking (Figure 6: AntPost). As a consequence, the clinical practice reduced the rectal NTCP, while the tracking approaches slightly increased the NTCP (Figure 7: $\triangle$ NTCP $=-0.06$ [Clinical], +0.03 $[\mathrm{MLC}],+0.01[\mathrm{MLC}+\mathrm{MU}])$. The MLC+MU tracking resulted in the planned boost dose, 335 while the MLC tracking increased the overall target dose (Figure 6). Nevertheless, the TCP differences were smaller than 0.01 , and the homogeneity errors were smaller than for the drift motion $(\Delta \mathrm{HI}=-2.7 \%$ [Static], 3.3\% [Clinical], $-5.7 \%[\mathrm{MLC}],-6.5 \%$ $[\mathrm{MLC}+\mathrm{MU}])$.

Hypo-fractionated: For a uniform hypo-fractionated treatment plan the clinical 340practice had a positioning error of the dose distribution in the anterior and superior direction (Figure 6) but the error was inside the PTV margin. An overall increase of the dose was measured for the MLC and MLC+MU tracking (Figure 6). The positioning error of the dose distribution as well as the increased dose of the tracking reduced the homogeneity $(\Delta \mathrm{HI}=-4.3 \%$ [Static], $-7.0 \%$ [Clinical], $-9.7 \%[\mathrm{MLC}],-8.0 \%[\mathrm{MLC}+\mathrm{MU}])$, 345but all TCPs were inside a 0.01 tolerance window (Figure 7: $\triangle T_{C P}$ Prostate \& Boost $<0.01$ ). The shift of the dose distribution with the clinical practice resulted in a NTCP reduction while the MLC and MLC+MU tracking increased these parameters (Figure 7: $\triangle N T C P=-0.10$ [Clinical], $0.21[\mathrm{MLC}], 0.15[\mathrm{MLC}+\mathrm{MU}])$.

\section{B.3. High Frequency prostate motion}

350Focal boost: The clinical practice delivered the dose distribution with a shift away from the rectum. This was corrected by the MLC and MLC+MU tracking. The MLC as well as the MLC+MU tracking had an increased dose posterior to the boost volume, with the 
smallest increase for the MLC+MU tracking. The MLC+MU tracking delivered the intended boost dose (DVH), but increased the CTV dose. The errors in the dose 355distribution did affect the homogeneity $(\Delta \mathrm{HI}=-2.8 \%$ [Static], $-3.7 \%$ [Clinical], $-7.1 \%$ $[\mathrm{MLC}],-6.9 \%[\mathrm{MLC}+\mathrm{MU}]$ ), and the rectal protection (Figure 7: $\Delta \mathrm{NTCP}=-0.07$ [Clinical], +0.08 [MLC], 0.01 [MLC+MU]). But the errors in the dose distribution did not affect the tumor control probability (Figure 7: $\Delta \mathrm{TCP}_{\text {Prostate }}=+0.01[\mathrm{MLC}],<0.01$ [Clinical, $\left.\mathrm{MLC}+\mathrm{MU}] ; \Delta \mathrm{TCP}_{\text {Boost }}<0.01\right)$.

360Hypo-fractionated: For a uniform hypo-fractionated treatment the MLC and MLC+MU tracking corrected an anterior-posterior positioning errosshift of the dose distribution in the anterior and superior direction whichas was observed for the clinical practice. However, the tracking increased the target dose. Both inconsistencies reduced the homogeneity $(\Delta \mathrm{HI}=-4.3 \%$ [Static], $-6.2 \%$ [Clinical], $-8.2 \%$ [MLC], $-8.9 \%$ $365[\mathrm{MLC}+\mathrm{MU}])$, but the errors were smaller than with the drift motion. Nevertheless, all TCPs were inside a 0.01 tolerance window (Figure 7). The shift of the dose distribution resulted in a reduced rectal NTCP while the tracking approaches increased thiese parameters (Figure 7: $\triangle \mathrm{NTCP}=-0.11$ [Clinical], $0.12[\mathrm{MLC}], 0.03[\mathrm{MLC}+\mathrm{MU}]$ ). 

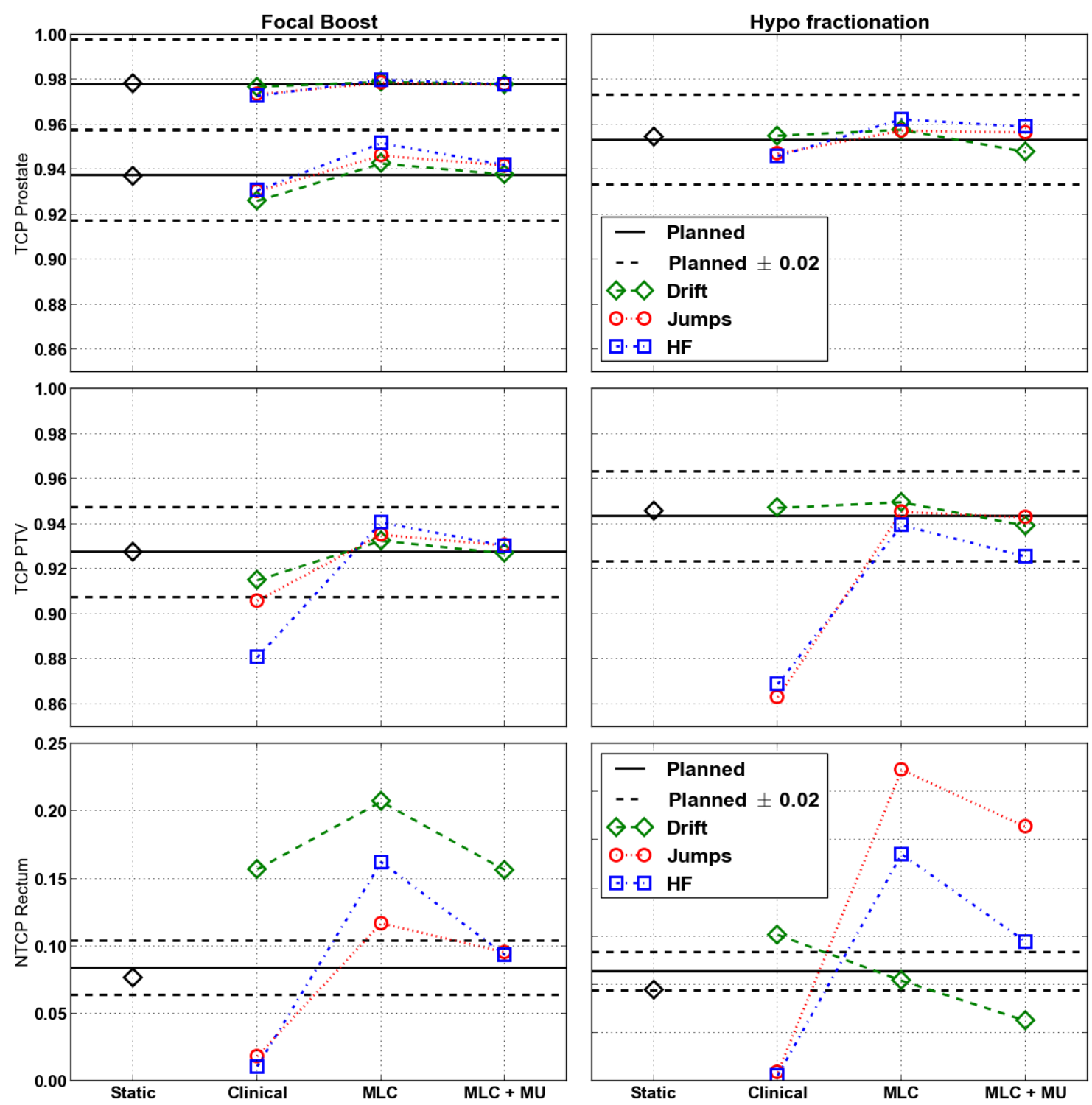

370Figure 7: Tumor control probabilities and normal tissue complication probabilities as measured using the 3D film-stack measurement.

\section{C. Treatment without PTV-margin}

Thus far the CTV to PTV margin made sure that the CTV received the intended dose (Figure 7: $\triangle T C P<0.01$ ) apart from two cases of the focal boost treatment: the clinical 375approach for the drift motion, and the MLC approach for the HF-motion $(\triangle T C P=-0.01)$. For a treatment without margin however, the clinical practice has a strongly reduced tumor control probability (Figure 7: PTV used as CTV). For such a treatment the MLC and MLC+MU tracking still result in the intended TCP (Figure 7: $\triangle \mathrm{TCP}<0.01$, except for two cases $+0.01[\mathrm{HF}$, focal boost, MLC], $-0.02[\mathrm{HF}$, hypo-fractionation, MLC+MU]). 


\section{Discussion}

\section{A. Proposed VMAT tracking}

Our previously proposed VMAT adaptation ${ }^{22}$ was designed to-allows for a real--time implementation because the correction processes $y$ usingare driven by only four fiducial points to drive all correction processes. The MU-correction for each control point could 385be calculated in $5.6 \mathrm{~ms}^{22}$. This is reasonable compared to the MLC latency for the current clinical VMAT tracking for prostate using a dedicated MLC tracking software (230 ms, Ref. ${ }^{3}$ ). The current method adds rotations and scaling in the MLC tracking, and MU-rescaling in the MLC+MU tracking.

The MLC and MLC+MU tracking delivered the dose distribution at the intended position, 390i.e. the position of the dose gradients (Figure 5 and Figure 6). While, a lack of control on this position can harm the surrounding normal tissues and critical organs. Additionally, the MLC+MU tracking resulted in the intended boost DVH in contrast to the MLC only tracking (e.g. Figure 6), and improved the rectal protection compared to MLC tracking (Figure 7). None of the methods (Clinical, MLC, MLC+MU) realized the intended control 3950 the rectal complication.

Both MLC and MLC+MU tracking increased the internal target dose (Figure 5 and Figure 6 , right panel) because the MLC could not fully achieve the required aperture resulting in a reducedintended MLC blocking inof the center of the beam (Figure 8). Such an increased target dose was also observed in the vicinity of the focal boost (Figure 6, left 400panel), where hich also had-a sharp dose gradient is used inside the CTV (similar to the dose gradient in Figure 5).

Ideally, the lack of blockingis could be solved by collimator (angle) tracking, i.e. correcting the rotational tracking component by adjusting theusing a collimator rotation such that the MLC motion is not rotated away from the modulation pattern set during 405initial optimization. Such an approach proved to be effective for our IMRT adaptation ${ }^{23}$. Constraints on the leaf motions ${ }^{19}$ during treatment planning could improve the results as well. 

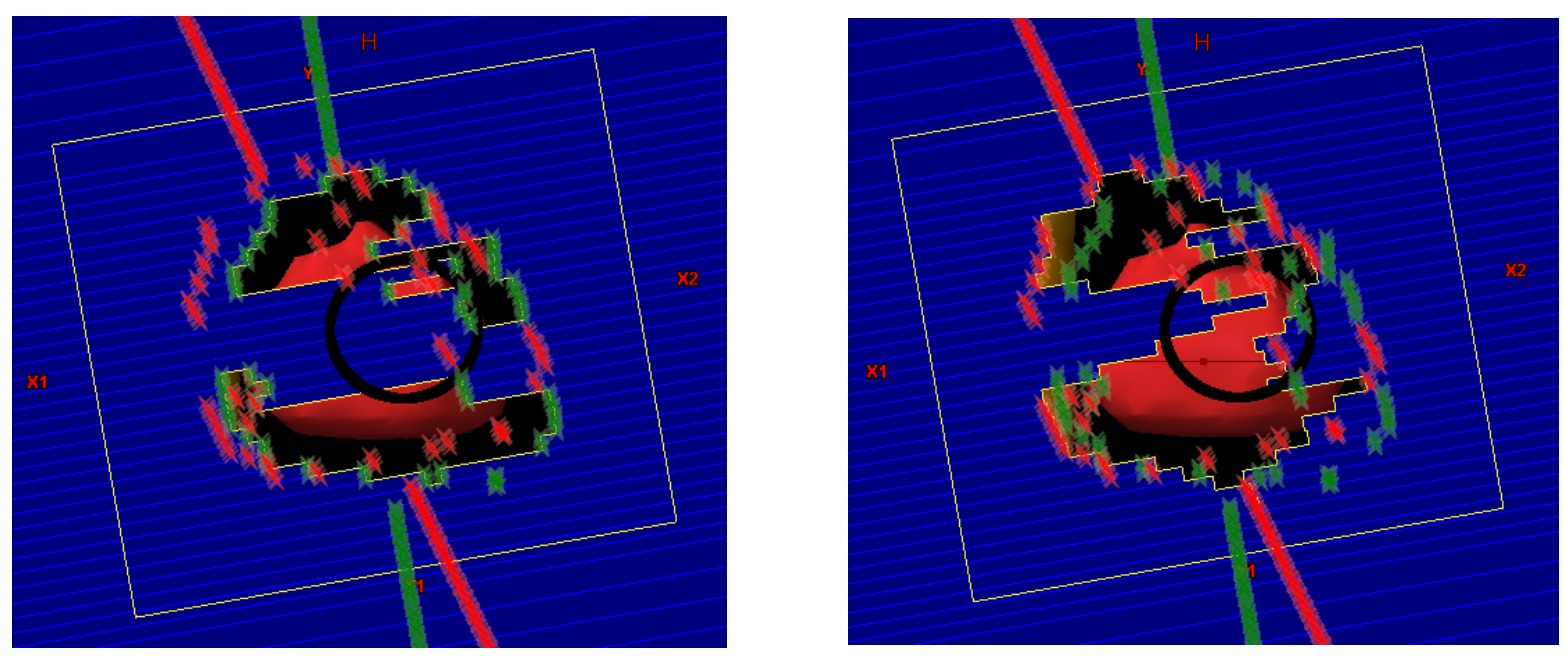

Figure 8: MLC blocking for CW10 of the hypo-fractionated plan at the moment when a persistent prostate Jump was introduced (Figure 5). The original MLC aperture is depicted on the left. The green 410 crosses correspond with this original aperture. After the required corrections the aperture should be as indicated by the red crosses. However the MLC is physically unable to realize these positions, resulting in the corrected MLC aperture in the right panel. Consequently, a reduced blocking of the area indicated by the black circle (right panel) is observed. The structures in the background are the initial rectal and prostate positions; these were depicted for orientation purposes only.

\section{B. Treatment planning}

For the tracking of breathing motion the MLC is often parallel to the longitudinal direction, which is the main breathing direction ${ }^{30,31}$ but also the gantry's rotation axis. For prostate the motion however, is mostly perpendicular to the gantry's rotation axis and such an optimal collimator angle is not feasible. Additionally, restricting the 420collimator angles reduces the degrees of freedom during treatment planning. Falk et al. introduced leaf position constraints ${ }^{19}$ during treatment planning simplifying the MLC modulation patterns, this which-could be beneficial for our tracking as well.

During treatment planning typically much attention is paid to the homogeneity of the target dose by requiring steep prostate DVHs (Figure 3). The static delivery already 425 showed a loss of these steep DVHs (Figure 3), and homogeneity $\left(\Delta \mathrm{HI}_{\text {Static }}=-2.8 \%\right.$ [focal boost] and $-4.3 \%$ [hypo-fractionation]). This effect is even worse when target motion is introduced. In contrast to these observations the boost DVH, planned with less strong constraints on the DVH, was perfectly achieved both with the static delivery and with the proposed MLC+MU tracking. 


\section{C. Experimental setup}

\section{C.1. Motion patterns}

The current phantom design did only allow coupled Anterior-Posterior and SuperiorInferior motion. Although this limitation reduces the probability of interplay effects, it is a realistic assumption for prostate motion ${ }^{21}$. The current study applied clinical prostate 435 motions of Ref. ${ }^{21}$, but the amplitude of the Jump and HF motions were scaled by a factor 0.58 and 0.67 respectively to remain within the phantom limits. Therefore, the presented errors in the position of the dose distribution shifts (Figure 5 and Figure 6) and resulting TCP PTV $_{\text {PT }}$ and rectal NTCP errors for the PTV and the rectum-(Figure 7 ) are an under estimation of clinical reality.

\section{C.2. Dose measurements}

\section{A major advantage of the current study is Fthe ability to use the same heterogeneous motion phantom for treatment planning and for two types of dose measurements perform-(IC and 3D film)-measurements in the same heterogeneous motion phantom as used for planning are a major advantage of the current study. But the 3D}

445measurements are cumbersome (Film measurements; cutting film, making a 3D stack, scanning in 2D, making a virtual 3D stack, etc.), and still a certain amount of dosimetric information is missing (only ten planes with a $4.6 \mathrm{~mm}$ separation). The film insert is designed to hold 12 film pieces, but the maximum height of the cut-away in the disk is already reached with 10 films. As a consequence, the measuring volume did not include 450the upper border of the high dose region. But this did not affect the prostate DVHs, see supplementary material. Additionally, the VOI covered only the upper half of the rectum. The rectal complication probabilities however, are strongly dependent on the maximal rectum dose and the part of the rectum receiving the maximal dose was included in the VOI.

\section{C.3. Advantages}

Our treatment units did not allow for a complete real-time tracking and a priori known motion trajectories were used. As such this work is limited to the plan-corrections themselves and not on the detection of marker motion. The synchronization approach has the advantages to allow both IC and film dose measurements of MLC and MLC+MU 460tracking; and it revealed the limitations of our current corrections while a complete implementation was avoided. 
Additionally, the current measurement setup allows a more detailed investigation of the proposed MLC+MU tracking e.g. the dosimetric impact/clinical relevance of latency (by adding phase shifts in the synchronization) and margin reduction (by applying different 465margins). Furthermore, the current setup allows an inter-comparison of different motion management strategies e.g. tracking (MLC or MLC+MU) vs. gating.

In our future work fiducials will be included in the phantom, and the phantom will be an essential tool for end-to-end quality assurance of the complete SBRT combination of motion management and modulated treatments i.e. motion detection and correction.

\section{D. Clinical relevance: is tracking needed?}

Despite an increased accuracy of the dose distribution using MLC and MLC+MU tracking (Figure 6) differences with the clinical practice were very small (Figure 7: $\triangle T C P_{\text {prostate \& Boost }}<0.02$ ) for both the focal boost treatment and the hypo-fractionation. The positioning error of the clinical practice reduced the rectal NTCP for two of the 475tested motions (Jumps, HF), but the Drift motion increased the NTCP. Although the bladder NTCP was not studied, the anterior shift of the dose distributions for the Jumps, and $\mathrm{HF}$ motion would probably increase the bladder NTCP. As a consequence it can only be concluded that the clinical practice was not able to realize the position of the dose deposition to conform to the one approved by the physician. The MLC and MLC+MU 480tracking improved the accuracy of the deposited dose distribution but did not improve the rectal protection either (Figure 7: NTCP).

Alternatively, a better organ at risk sparing can also be achieved by reducing the required high dose volume (reducing margins), which was evaluated by considering the PTV as a zero margin CTV (Figure 7: PTV). Here the positioning error of the dose 485distribution with the clinical practice reduced the TCP, which had the largest impact for the hypo-fractionation scheme. This is due to the dependence of the tumor control on the minimal dose received.

For the boost volume in the focal boost treatment this minimal dose is always at least the CTV dose. Therefore, the TCP values of the boost volume are not impacted by the 490selection of any of the methods (Clinical, MLC, MLC+MU). Notwithstanding this, only the MLC+MU tracking was able to deposit the intended boost dose, which was planned without margins (Figure 6). Therefore, conclusions of studies using in-homogeneous 
dose distribution without tracking will be hampered by the large dose uncertainties. When inhomogeneous dose distributions would be used to locally reduce the dose (e.g. 495to protect the uretra) clinically relevant effects (NTCP-changes) might be expected because organ protection is highly dependent on the maximal received dose. 


\section{Conclusion}

Dosimetric (MLC+MU) tracking was compared to a geometric (MLC) tracking and our clinical practice (no tracking) for both a non-uniform focal boost and a hypo500fractionation planning approach. Treatment planning, IC measurements, and 3D filmstack measurements were performed on the same heterogeneous prostate motion phantom, which applied three realistic internal phantom motions to the prostate rather than whole phantom motion.

Static measurements showed a reduced homogeneity of the target dose, which $505 q u e s t i o n s$ the current focus on target homogeneity during treatment planning. Adding internal patient/phantom motion further blurs the dose distribution. The boost DVH, with less steep dose gradients, could be deposited by the MLC+MU tracking despite a lack of blocking caused by an interplay between the MLC modulations and the MLC corrections. A further improvement of the current technique could be using the 510collimator angle to account for target rotations (collimator tracking).

The current clinical practice achieved the intended tumor control probabilities for all targets, because of the used PTV margins and the high doses used (35x2.2 Gy and $35 \times 2.7$ Gy [focal boost], 5x7.25 Gy [hypo-fractionation]). However, the dose profiles showed a positioning error of the measured dose distributions, which largely impacts 515the rectal complication probability. In the current study this impact was in favor of the rectum in two out of three cases. But, a negative impact is not excluded.

The MLC and the MLC+MU tracking based on the info from four fiducial points only, was sufficient to increase control on the position of the delivered dose distributions. Only the MLC+MU tracking was able to deposit a dose distribution with the intended boost DVH. 520 Nevertheless, the improved target dose had only limited impact on the tumor control probability (TCP differences $<0.02$ ).

Both the current clinical practice and the current MLC tracking have large dose uncertainties $(10 \%)$ which can be of relevance in clinical studies involving non-uniform dose distributions. The current clinical control is caused by the CTV to PTV margins; a 525further reduction of treatment margins would require a motion management like the MLC or the MLC+MU tracking. 


\section{Acknowledgements}

The group of Prof. P. Keall for sharing the prostate motion files.

Research partly funded by Varian Medical Systems, Palo Alto, California, USA. 


\section{Bibliography}

A. Schweikard and G. Glosser, "Robotic motion compensation for respiratory movement during radiosurgery," J. Comput. Surg. 102, (2000).

T. Depuydt, K. Poels, D. Verellen, B. Engels, C. Collen, C. Haverbeke, T. Gevaert, N. Buls, G. Van Gompel, T. Reynders, M. Duchateau, K. Tournel, M. Boussaer, F. Steenbeke, F. Vandenbroucke, and M. De Ridder, "Initial assessment of tumor tracking with a gimbaled linac system in clinical circumstances: a patient simulation study.," Radiother. Oncol. 106(2), 236-40 (2013).

P.J. Keall, E. Colvill, R. O'Brien, J.A. Ng, P.R. Poulsen, T. Eade, A. Kneebone, and J.T. Booth, "The first clinical implementation of electromagnetic transponder-guided MLC tracking.," Med. Phys. 41(2), 020702 (2014).

M.F. Fast, S. Nill, J.L. Bedford, and U. Oelfke, "Dynamic tumor tracking using the Elekta Agility MLC.," Med. Phys. 41(11), 111719 (2014).

J. Adamson, Q. Wu, and D. Yan, "Dosimetric effect of intrafraction motion and residual setup error for hypofractionated prostate intensity-modulated radiotherapy with online cone beam computed tomography image guidance.," Int. J. Radiat. Oncol. Biol. Phys. 80(2), 453-61 (2011).

T.R. Mackie, "Tomotherapy: A new concept for the delivery of dynamic conformal radiotherapy," Med. Phys. 20(6), 1709 (1993).

K.M. Langen, W. Lu, T.R. Willoughby, B. Chauhan, S.L. Meeks, P. a Kupelian, and G. Olivera, "Dosimetric effect of prostate motion during helical tomotherapy.," Int. J. Radiat. Oncol. Biol. Phys. 74(4), 1134-42 (2009).

K.M. Langen, B. Chauhan, J. V Siebers, J. Moore, and P. a Kupelian, "The dosimetric effect of intrafraction prostate motion on step-and-shoot intensity-modulated radiation therapy plans: magnitude, correlation with motion parameters, and comparison with helical tomotherapy plans.," Int. J. Radiat. Oncol. Biol. Phys. 84(5), 1220-5 (2012).

S. Arcangeli, M. Scorsetti, and F. Alongi, "Will SBRT replace conventional radiotherapy in patients with low-intermediate risk prostate cancer? A review," Crit. Rev. Oncol. Hematol. 84(1), 101-108 (2012).

a. Katz, D. Freeman, J. Aronovitz, D. Fuller, G. Bolzicco, R. Meier, S. Collins, J. Wang, M. Steinberg, and C. King, "Five-year Biochemical Control Rates for Stereotactic Body Radiation Therapy for Organ-confined Prostate Cancer: A Multi-institutional Pooled Analysis," Int. J. Radiat. Oncol. 84(3), S147-S148 (2012).

A. Loblaw, P. Cheung, L. D'Alimonte, A. Deabreu, A. Mamedov, L. Zhang, C. Tang, H. Quon, S. Jain, G. Pang, and R. Nam, "Prostate stereotactic ablative body radiotherapy using a standard linear accelerator: Toxicity, biochemical, and pathological outcomes," Radiother. Oncol. 107(2), 153-158 (2013). 
a. C. Tree, E.J. Alexander, N.J. Van As, D.P. Dearnaley, and V. Khoo, "Biological dose escalation and hypofractionation: What is there to be gained and how will it best be done?," Clin. Oncol. 25(8), 483-498 (2013).

$570^{13}$ I.M. Lips, U.A. van der Heide, K. Haustermans, E.N.J.T. van Lin, F. Pos, S.P.G. Franken, A.N.T.J. Kotte, C.H. van Gils, and M. van Vulpen, "Single blind randomized phase III trial to investigate the benefit of a focal lesion ablative microboost in prostate cancer (FLAME-trial): study protocol for a randomized controlled trial.," Trials 12(1), 255 (2011).

$575^{14}$ K. Otto, "Volumetric modulated arc therapy: IMRT in a single gantry arc," Med. Phys. 35(1), 310-317 (2008).

E. Colvill, P.R. Poulsen, J.T. Booth, R.T. O'Brien, J. a Ng, and P.J. Keall, "DMLC tracking and gating can improve dose coverage for prostate VMAT.," Med. Phys. 41(9), 091705 (2014).

$580^{16}$ P. Poulsen, M. Schmidt, and P. Keall, "A method of dose reconstruction for moving targets compatible with dynamic treatments," Med. Phys. 39(October), 6237-6246 (2012).

M. Falk, T. Pommer, P. Keall, S. Korreman, G. Persson, P. Poulsen, and P. Munck af Rosenschöld, "Motion management during IMAT treatment of mobile lung tumors--a comparison of MLC tracking and gated delivery.," Med. Phys. 41(10), 101707 (2014).

H.S. Li, I.J. Chetty, C. a Enke, R.D. Foster, T.R. Willoughby, P. a Kupellian, and T.D. Solberg, "Dosimetric consequences of intrafraction prostate motion.," Int. J. Radiat. Oncol. Biol. Phys. 71(3), 801-12 (2008).

M. Falk, T. Larsson, P. Keall, B. Chul Cho, M. Aznar, S. Korreman, P. Poulsen, and P. Munck Af Rosenschold, "The dosimetric impact of inversely optimized arc radiotherapy plan modulation for real-time dynamic MLC tracking delivery.," Med. Phys. 39(3), 1588-94 (2012).

P.R. Poulsen, W. Fledelius, B. Cho, and P. Keall, "Image-based dynamic multileaf collimator tracking of moving targets during intensity-modulated arc therapy.," Int. J. Radiat. Oncol. Biol. Phys. 83(2), e265-71 (2012).

K.M. Langen, T.R. Willoughby, S.L. Meeks, A. Santhanam, A. Cunningham, L. Levine, and P. a Kupelian, "Observations on real-time prostate gland motion using electromagnetic tracking.," Int. J. Radiat. Oncol. Biol. Phys. 71(4), 1084-90 (2008). 3891 (2015).

W. Crijns, H. Van Herck, G. Defraene, L. van den Bergh, P. Slagmolen, K. Haustermans, F. Maes, F. Van den Heuvel, H. Van Herck, L. Van Den Bergh, and F. Van Den Heuvel, "Dosimetric adaptive IMRT driven by fiducial points," Med. Phys. 41(6), 061716-1 - 11 (2014). 
W. Crijns, F. Maes, U. a van der Heide, and F. Van den Heuvel, "Calibrating page sized Gafchromic EBT3 films.," Med. Phys. 40(1), 012102 (2013).

S. Webb and A.E. Nahum, "A model for calculating tumour control probability in radiotherapy including the effects of inhomogeneous distributions of dose and clonogenic cell density," Phys. Med. Biol. 38(6), 653-656 (1993).

M. Oldham, A. Neal, and S. Webb, "A comparison of conventional 'forward planning' with inverse planning for 3D conformal radiotherapy of the prostate.," Radiother. Oncol. 35(3), 248-62 (1995).

27 J.T. Lyman, "Complication probability as assessed from dose-volume histograms.," Radiat. Res. Suppl. 8, S13-9 (1985).

G.J. Kutcher and C. Burman, "Calculation of complication probability factors for nonuniform normal tissue irradiation: The effective volume method gerald," Int. J. Radiat. Oncol. ... 16(December), 1623-1630 (1989).

J.M. Michalski, H. Gay, A. Jackson, S.L. Tucker, and J.O. Deasy, "Radiation dosevolume effects in radiation-induced rectal injury.," Int. J. Radiat. Oncol. Biol. Phys. 76(3 Suppl), S123-9 (2010).

P.J. Keall, A. Sawant, B. Cho, D. Ruan, J. Wu, P. Poulsen, J. Petersen, L.J. Newell, H. Cattell, and S. Korreman, "Electromagnetic-guided dynamic multileaf collimator tracking enables motion management for intensity-modulated arc therapy.," Int. J. Radiat. Oncol. Biol. Phys. 79(1), 312-20 (2011).

P.R. Poulsen, B. Cho, D. Ruan, A. Sawant, and P.J. Keall, "Dynamic multileaf collimator tracking of respiratory target motion based on a single kilovoltage imager during arc radiotherapy.," Int. J. Radiat. Oncol. Biol. Phys. 77(2), 600-7 (2010). 
630Supplementary material:

Potential benefits of dosimetric VMAT tracking verified with 3D film dose measurements

\section{Synchronizing the phantom motion and the treatment unit}

Our current treatment unit did not allow for real-time tracking. Therefore, generation of 635the corrected treatment plans was performed in preparation of the experiments using the known motion patterns (i.e. not real-time), but the actual dose measurements were performed using the same motion patterns, see flow chart in the main manuscript (Figure 2). This setup required a synchronization of the treatment unit and the motion phantom.

640The synchronization was obtained by putting the treatment unit in "gating mode", allowing an immediate response (beam-on) of the treatment unit when an external marker block exceeds a predefined threshold. The external marker block was positioned on the dedicated surrogate motion driver of the phantom, which is synchronized with the internal phantom motion. Next, a surrogate square wave motion was programmed 645such that the trigger exceeds the threshold at time points 21s, 116s, 211s, and 306s.

The first $21 \mathrm{~s}$ allowed checking the setup (Figure 2 in the main manuscript). The 95s between two succeeding arcs included 60 s of beam-on, and 35 s to change the beam settings (e.g. collimator angle). The total irradiation time of each arc was not altered by the tracking procedure or the actual dose delivery. Consequently, synchronization errors 650 were certainly smaller than $0.6 \mathrm{~s}$ (read out precision). A possible remaining latency of the treatment unit was ignored.

\section{Film measurements}

For each film measurement a single sheet of EBT3 ${ }^{f}$ was used. The films were cut with a laser cutter $^{g}$, Figure A.1. The resulting film pieces perfectly fit in the dedicated film 655insert. An orientation slit was cut in the film to verify the correct film orientation during irradiation and read out. For read out, the films pieces were puzzled in the original order

f Ashland Specialty Ingredients, Wayne, New Jersey

g FabLab, Heverlee, Belgium 
to be scanned as a single image. This image was converted to a 2D dose image according to our film dosimetry protocol ${ }^{24}$.

Next, the 2D dose image was split into the different dose planes. To reduce positioning 660 errors during the scanning procedure, each dose plane was rigidly registered with a template using the assembling guiding holes as registration features. The registered digital dose planes were stacked using a plane separation of $4.6 \mathrm{~mm}$.

The resulting measurement's coordinate system was rotated compared to the TPScoordinate system ( $46^{\circ}$ around the left-right axis, see Figure 1 in the main text). To 665 cope with this different orientation the dose data with the lowest plane separation (TPS) was reformatted towards the dose data with the highest plane separation using a trilinear interpolation in MevisLab ${ }^{\mathrm{h}}$. Although this film stack allowed a measurement in three dimensions, there was a loss of information due to limited volume of interest (VOI) and sparse sampling in the cross-plane direction, see Figure A.1. For the prostate 670 no effect was observed. But the limited VOI had an impact on the rectal DVH (Figure A.1, green arrow). The rectal complications were mainly caused by the maximal dose to the organ; this area was covered by the VOI. For the small boost volume the plane separation had a blurring effect (Figure A.1, blue arrow). All comparisons presented in the work were based on dose distributions in the same coordinates system and with the 675 same resolution, i.e. the measurement's coordinate system.

Statements on the location of the dose distribution were supported by a qualitative analysis of line profiles rather than the DVH analysis which may be impacted by changing volume-surface ratios.

h MeVis Medical Solutions AG, Germany 

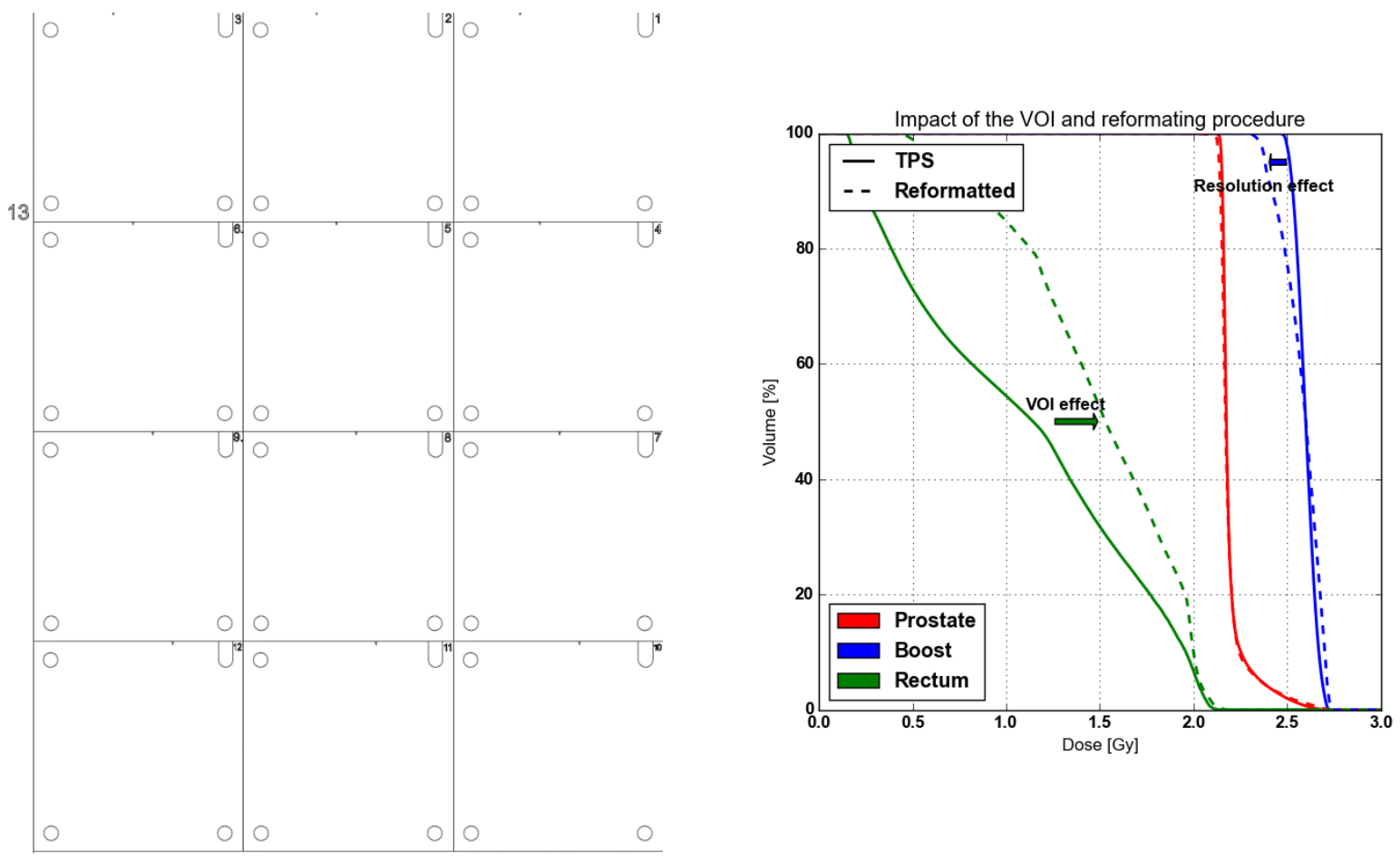

Figure A.1: Left: Input file of the laser cutter. Each film piece has four holes which perfectly fit around 680the guides of the film stack. Right: Impact of the VOI and the reformatting of the TPS calculated 3D dose distribution towards the measurement's coordinate system for the initial focal boost plan. 